Voix et Images

volxetimages

\title{
Imbrications du récit contemporain
}

\section{François Paré}

Volume 31, numéro 2 (92), hiver 2006

Gilles Archambault

URI : https://id.erudit.org/iderudit/012882ar

DOI : https://doi.org/10.7202/012882ar

Aller au sommaire du numéro

Éditeur(s)

Université du Québec à Montréal

ISSN

0318-9201 (imprimé)

1705-933X (numérique)

Découvrir la revue

Citer cet article

Paré, F. (2006). Imbrications du récit contemporain. Voix et Images, 31(2),

149-152. https://doi.org/10.7202/012882ar d'utilisation que vous pouvez consulter en ligne.

https://apropos.erudit.org/fr/usagers/politique-dutilisation/ 


\author{
E S S A IS / É T U D ES \\ Imbrications du récit contemporain \\ $++$
}

FRANÇOIS PARÉ

Université de Waterloo

Deux ouvrages collectifs, élaborés à partir des travaux d'une équipe interuniversitaire de recherche sur la dynamique des genres en littérature québécoise contemporaine, feront l'objet de cette chronique. Réunissant les textes d'une trentaine d'universitaires, ces deux parutions d'envergure s'intéressent aux éléments narratifs qui pénètrent assez régulièrement aujourd'hui la poésie, l'essai, le roman et le théâtre, contribuant au décloisonnement de ces genres littéraires. L'entreprise présentée sur plus de 600 pages recoupe l'ensemble de la production romanesque et thêâtrale des vingt-cinq dernières années au Québec et offre l'impressionnant panorama d'une littérature étonnamment vivante et d'une grande diversité thématique et formelle. Avatars de programmes subventionnaires favorisant les projets facilement comptabilisables, les deux ouvrages recensent pas moins de 150 récits et pièces de théâtre d'importance très variable. Il en résulte un inévitable effet de survol et surtout une recherche assidue des points de convergence entre ces textes fort inégaux. Il est rare d'y voir une œuvre analysée pour sa dynamique intrinsèque (surtout dans le premier volume), les auteurs cherchant plutôt à y repérer les signes - parfois superficiels - de la problématique à l'étude. Crédit est donné heureusement aux auxiliaires de recherche qui ont dû passer des heures à dépouiller ce corpus quelque peu excessif!

Le premier ${ }^{1}$ de ces deux ouvrages porte sur la coexistence et l'imbrication des éléments narratifs dans la poésie, le roman et l'essai québécois depuis 1980 environ. Constatant que la littérature contemporaine exige une traversée des genres et un réexamen du discours narratif, René Audet et Andrée Mercier expliquent dans leur introduction les objectifs généraux du recueil qu'ils dirigent: "[V]oir comment le geste du raconter investit (à divers niveaux, sous diverses formes) l'ensemble des

1 René Audet et Andrée Mercier (dir.), La narrativité contemporaine au Québec, t. I. La littérature et ses enjeux narratifs, Québec, Les Presses de l'Université Laval, 2004, 314 p. 
pratiques littéraires.» (7) La narrativité est définie, un peu à la manière de Paul Ricœur, comme «l'inscription d'événements dans le temps, selon un ordre ou une configuration particulière» (9). Cette définition permet de mieux saisir la prise en charge de la fiction par un sujet écrivant. La narrativité n'est donc pas tant un produit de la lecture que nous en faisons qu'une intention scripturale qui mobilise l'œuvre dès sa conception. Malgré la diversité des auteurs et des corpus étudiés, cette définition claire et très utile assure la cohérence exceptionnelle de l'ouvrage.

Plusieurs auteurs notent l'éclatement du tissu fictionnel dans le roman québécois contemporain. Si narrativité il y a, c'est sous l'angle du fragment et de la mise en recueil qu'il convient de l'aborder. Dans un article qui sert à la fois d'introduction théorique et de mise en contexte, René Audet et Thierry Bissonnette évaluent l'impact de l'imbrication du narratif chez certains poètes, essayistes et romanciers, tels Jacques Brault, Élisabeth Vonarburg et Suzanne Jacob. S'il se déploie le plus souvent selon le mode d'une "narrativité séquentielle», le recueil de poèmes suspend la logique habituelle du récit, notamment la relation causale. Quant à l'essai, Audet et Bissonnette estiment qu'il est souvent accompagné d'exemples et de faits divers dont les éléments narratifs sont évidents. Ils taisent cependant la composante autobiographique, éminemment présente dans la pratique de l'essai au Québec.

Dans une autre étude, Anne-Marie Clément se concentre sur l'expression de la discontinuité dans le récit contemporain, notamment chez Paul Chanel Malenfant, Jacques Poulin et Élise Turcotte, une perspective qu'adopte également Michel Lord dans son étude des nouvelles de Diane-Monique Daviau. Dans cette dernière analyse, Lord explore la notion très intéressante du «descriptif narrativisé»; ces formes circulaires, si souvent à l'œuvre chez Daviau, interviennent «lorsque le discours présente des complications très fortes, qu'il se perd alors dans les tourbillons, les volutes, les méandres, les dédales d'un onirisme des plus étranges» (253). C'est également à la nouvelle que s'intéresse Denis Sauvé dans son étude de l'accélération et de la lenteur chez Gaétan Brulotte et André Brochu. Le texte bref alimenterait chez le lecteur une tension jouissive entre "le désir d'atteindre la fin au plus vite » et celui de retarder l'échéance promise par la concision du genre.

Au Québec, comme ailleurs, de nombreux romanciers ont cherché à entrecouper la succession des événements racontés d'une dimension essayistique par laquelle s'exprime une pensée spéculative. Cette technique n'est pas neuve: bien des textes médiévaux faisaient appel au discours réflexif, ne serait-ce que pour situer l'œuvre dans son environnement moral ou religieux. Robert Dion, dans une très belle étude, ne s'étonne donc pas de retrouver des formes hybrides entre l'essai et le roman chez des écrivains aussi différents que Gérard Bessette, Marco Micone et Régine Robin. Les formes de l'essai sont parfois si envahissantes dans ces textes que la fiction $s^{\prime} y$ trouve constamment repoussée comme si elle était un élément indésirable, gênant la lecture et l'interprétation du récit. Par ailleurs, Andrée Mercier et Frances Fortier recensent la «logique du sensible» dans de nombreux récits où les auteurs ont cherché non pas à raconter des événements, mais à « documenter toutes les facettes de la narration» (196). 
Il ne faudrait pas omettre enfin le chapitre important que consacre Élisabeth Haghebaert à l'œuvre récente de Réjean Ducharme. Dans la dynamique délinquante du langage mise en jeu par l'auteur de Dévadé, de Va savoir et de Gros mots, peutêtre sommes-nous en présence des formes les plus transversales de la narrativité, celles qui s'accommodent de peu. Ce sont ces «simulacres» du langage qui, chez Ducharme, marquent la surprésence du texte et l'éviction de son sujet central.

Dans Le thêâtre et ses nouvelles dynamiques narratives ${ }^{2}$, Chantal Hébert et Irène Perelli-Contos nous invitent à examiner l'intrusion d'éléments narratifs dans la dramaturgie québécoise depuis le début des années 1980. L'ouvrage qui en résulte offre une perspective éminemment originale sur l'ensemble de la production théâtrale contemporaine, du thêâtre des femmes aux soliloques de Larry Tremblay et de Daniel Danis. Moins portées à légitimer de vastes corpus, les études rassemblées se concentrent sur l'évolution du texte scénique et sur les éléments particuliers qui témoignent de la «dimension visuelle » et de la "matérialité du langage » au théâtre (17-18). Je me contenterai de rendre compte des études qui traitent de la dramaturgie québécoise. Certains chapitres de ce livre (notamment ceux de Denyse Noreau sur Vladimir Nabokov et de Joseph Danan sur Bernard-Marie Koltès et Valère Novarina) ne répondent guère aux objectifs de l'ouvrage et auraient dû être retranchés.

Ce qui constitue le cœur des études rassemblées ici, c'est la "contamination " du dialogue théâtral par un récit de soi envahissant, témoignant «d'un sujet en crise, en état de faiblesse intérieure» (173). Marie-Christine Lesage et Adeline Gendron s'interrogent, par exemple, sur l'insertion d'éléments narratifs dans le texte de theâtre contemporain. Elles font remarquer que, dans les pièces monologiques de Larry Tremblay, les structures narratives hybrides s'apparentent au soliloque, «forme qui épouse les contours de la subjectivité et de l'espace intime du personnage» (195). C'est dans l'article d'Irène Roy que l'on peut trouver l'armature théorique permettant de mieux comprendre l'imbrication des formes du récit dans le thêâtre québécois contemporain. Roy constate que l'espace public tout entier tend à accueillir aujourd'hui "l'expression monologique de la personnalité» (44). Si, au même moment, le monologue a pu envahir le théâtre, c'est que la scène était particulièrement apte à servir de réceptacle métaphorique pour le témoignage personnel et le récit intime.

Dans un texte magistral, Lucie Robert retrace pour sa part la présence du "grand récit féminin» dans le théâtre des femmes. Remontant aux monologues du «corps à corps avec la mère» dans les œuvres de Pol Pelletier et de Louisette Dussault au tournant des années 1980, Robert évoque l'invention d'un sujet féminin issu non pas des tensions dramatiques entre les personnages, mais de la narrativité quasi thérapeutique autorisée par la parole directe adressée aux spectateurs. Enfin,

2 Chantal Hébert et Irène Perelli-Contos (dir.), La narrativité contemporaine au Québec, t. II. Le théâtre et ses nouvelles dynamiques narratives, Québec, Les Presses de l’Université Laval, 2005, 313 p. 
dans le même ordre d'idées, Katherine Papachristos cherche à définir l'espèce $\mathrm{d}^{\prime}$ " égarement» qui frappe les personnages de Daniel Danis au terme de leur voyage imaginaire dans l'oubli : «[C]et égarement se traduit par la retraite des personnagesnarrateurs dans les lieux du souvenir (plutôt des non-lieux) qui provoquent les tirades rétrospectives de leurs vies passées.» (201) Par sa théâtralisation du langage, Danis impose au théâtre, selon Papachristos, une matérialité nouvelle, liée à une oralité rendue purement visible.

Sur cette même question, Yves Jubinville examine les répercussions du textetémoignage sur la dramaturgie elle-même, car ne faut-il pas pour la première fois «élaborer un dispositif de production et de réception de la parole intime" (43)? Jubinville conclut que l'émergence du témoignage au théâtre s'inscrit pleinement dans l'effort de comprendre le rôle de l'intime dans la sphère publique et de promouvoir une vision du sujet en interaction avec le monde sensible.

Enfin, rejoignant l'intérêt du premier volume sur la narrativité dans le récit, Andrée Mercier, Denise Cliche et Isabelle Tremblay consacrent une très belle étude à l'œuvre de Carole Fréchette, dont le thêâtre fait une place importante à la fragmentation sous toutes ses formes, celle du tissu énonciatif de l'œuvre autant que du «morcellement d'un corps qui n'en finit plus d'énumérer ses parties» (218). En effet, c'est dans des œuvres-maîtresses de la dramaturgie québécoise contemporaine, telles Baby blues et Les quatre morts de Marie, que s'affirme justement la présence d'une narrativisation assez particulière de la scène, alors que le thêâtre n'arrive plus tout à fait à médiatiser le corps des comédiens sans un recours stratégique aux «caractéristiques sensibles de la présence» (241).

Dans l'ensemble, la lecture de ces deux volumes rend donc tout à fait compte de ce merveilleux outil de renouvellement et de transformation de la littérature et du théâtre qu'est le récit lui-même. La richesse de la littérature québécoise est remarquable à ce titre. La présence soutenue du récit, au-delà des marqueurs génériques et au croisement de l'homogène et de l'hétérogène, confirme hors de tout doute la survivance des grandes pulsions narratives au sein des sociétés actuelles. 